\title{
ON CURVATURE HOMOGENEOUS AND LOCALLY HOMOGENEOUS AFFINE CONNECTIONS
}

\author{
BARBARA OPOZDA \\ (Communicated by Christopher Croke)
}

\begin{abstract}
This paper deals with curvature homogeneous affine connections on 2-dimensional manifolds. We give a sufficient condition for a projectively flat curvature homogeneous connection to be locally homogeneous and show how to construct curvature homogeneous connections that are not locally homogeneous.
\end{abstract}

The concept of a curvature homogeneous Riemannian connection was introduced by I. M. Singer in [5] and then studied in many papers. A similar notion can be defined in affine geometry. Curvature homogeneous affine connections do not have, in general, properties similar to Riemannian ones. For instance, a curvature homogeneous Riemannian connection on a 2-dimensional manifold is automatically locally symmetric and consequently locally homogeneous. As regards the affine case, it is easy to find curvature homogeneous affine connections on 2-dimensional manifolds which are not locally homogeneous. For instance, connections with symmetric Ricci tensor of rank 1 on connected manifolds are always curvature homogeneous and only in exceptional cases locally homogeneous. In this note we begin to study curvature homogeneous affine connections on 2-dimensional manifolds. The differences between the curvature homogeneity, locally homogeneity and local symmetry conditions are illustrated by means of projectively flat connections, which, in some sense, are the closest to locally symmetric ones.

1

Manifolds are assumed to be connected and connections torsion-free. For a given connection its curvature and Ricci tensors will be denoted by $R$ and Ric respectively.

Definition 1.1. Let $\nabla$ be a connection on a manifold $M$. It is called curvature homogeneous of order $r$ if and only if for every $x, y \in M$ there exists a linear isomorphism $F: T_{x} M \rightarrow T_{y} M$ such that $F^{*}\left(\nabla^{i} R\right)_{y}=\left(\nabla^{i} R\right)_{x}$ for every $i=0, \ldots, r$. By a curvature homogeneous connection we mean a connection which is curvature homogeneous of order 0.

Received by the editors November 15, 1994.

1991 Mathematics Subject Classification. Primary 53B05, 53C30.

Key words and phrases. Affine connections: locally homogenous, curvature homogeneous, projectively flat, locally symmetric.

The research was partially supported by the KBN grant no. 2 P301 03004. 
If $M$ is a 2-dimensional manifold, then the Ricci tensor determines the curvature tensor. Namely $R(X, Y) Z=\operatorname{Ric}(Y, Z) X-\operatorname{Ric}(X, Z) Y$. Therefore, in this case, $\nabla^{i} R$ can be replaced by $\nabla^{i} R i c$ in the above definition.

Definition 1.2. An affine connection on a manifold $M$ is locally homogeneous if and only if for every $x, y \in M$ there are neighbourhoods $U$ of $x$ and $V$ of $y$, and an affine transformation $f:\left(U, \nabla_{\mid U}\right) \rightarrow\left(V, \nabla_{\mid V}\right)$ sending $x$ into $y$.

A connection is locally symmetric if and only if $\nabla R=0$. It is clear that a locally symmetric connection is locally homogeneous and a locally homogeneous connection is curvature homogeneous of any order. On a 2-dimensional manifold a Ricci-symmetric connection is projectively flat if and only if $\nabla \operatorname{Ric}(X, Y, Z)$ is symmetric in all arguments. In what follows the assumption that $\nabla$ is projectively flat will automatically include the assumption that Ric is symmetric. Let us also point out that on a 2-dimensional manifold a connection is Ricci-symmetric if and only if it is semi-symmetric, i.e. $R_{X Y} \cdot R=0$.

In this section we shall consider projectively flat connections whose Ricci tensor has rank 1 and ker Ric is parallel. Such connections naturally appear on nondegenerate affine minimal ruled surfaces as well as on cylinders in $\mathbf{R}^{3}$; see [2], [3], [4]. We shall show that even under such strong assumptions about the connections most of them are not locally homogeneous though all of them are curvature homogeneous.

The following information will be useful. For a proof see [2], [3].

Lemma 1.1. Let $\nabla$ be a Ricci-symmetric connection on a 2-dimensional manifold and rk Ric $=1$ on $M$. The distribution ker Ric is $\nabla$-parallel if and only if for every $x \in M$ there is a coordinate system $(u, v)$ on some neighbourhood $U$ of $x$ such that $\partial_{u}$ spans ker Ric at each point of $U$ and

$$
\nabla_{\partial_{u}} \partial_{u}=0, \quad \nabla_{\partial_{u}} \partial_{v}=C \partial_{u}, \quad \nabla_{\partial_{v}} \partial_{v}=A \partial_{u}
$$

for some functions $C$ and $A$, where $C_{u}=0$. Moreover we have on $U$ :

$$
\nabla R i c \text { is symmetric in all arguments if and only if } \iota_{u}=0 \text {, }
$$

where $\iota=\operatorname{Ric}\left(\partial_{v}, \partial_{v}\right)=A_{u}-C_{v}-C^{2}$.

A coordinate system described in the above lemma will be called canonical for $\nabla$. A canonical coordinate system is not unique around a fixed point.

Theorem 1.2. Let $\nabla$ be an analytic projectively flat connection on a 2-dimensional analytic manifold $M$. Assume that the Ricci tensor of $\nabla$ has rank 1 and the distribution ker Ric is $\nabla$-parallel. If $\nabla$ is curvature homogeneous of order 1 , then it is locally homogeneous.

Proof. If $(u, v)$ is a canonical coordinate system for $\nabla$, then the only non-zero terms of $\nabla^{k}$ Ric for $k=0,1, \ldots$ might be $\nabla^{k} \operatorname{Ric}\left(\partial_{v}, \ldots, \partial_{v}\right)=\iota^{(k)}$, where $\iota=\operatorname{Ric}\left(\partial_{v}, \partial_{v}\right)$ is a function of $v$.

Let $x_{o}$ be a fixed point and $(\tilde{u}, \tilde{v})$ be a canonical coordinate system around $x_{o}=\left(\tilde{u}_{o}, \tilde{v}_{o}\right)$. Let $\tilde{\iota}=\operatorname{Ric}\left(\partial_{\tilde{v}}, \partial_{\tilde{v}}\right)$. For the sake of simplicity we shall write $\iota_{o}=\tilde{\iota}_{x_{o}}$, 
$\iota_{o}^{\prime}=\tilde{\iota}_{x_{o}}^{\prime}$ and $\iota_{o}^{(k)}=\tilde{\iota}_{x_{o}}^{(k)}$ for $k=0,1, \ldots$ Take a canonical coordinate system around another point. For every $x$ from the domain $U$ of $(u, v)$ there is a linear mapping $F_{x}: T_{x_{o}} M \rightarrow T_{x} M$ such that

$$
F_{x}\left(\partial_{\tilde{u}}\right)_{x_{o}}=a\left(\partial_{u}\right)_{x}, \quad F_{x}\left(\partial_{\tilde{v}}\right)_{x_{o}}=b\left(\partial_{u}\right)_{x}+d\left(\partial_{v}\right)_{x}
$$

and

$$
\begin{aligned}
& \operatorname{Ric}_{x}\left(F_{x}\left(\partial_{\tilde{v}}\right)_{x_{o}}, F_{x}\left(\partial_{\tilde{v}}\right)_{x_{o}}\right)=\iota_{o}, \\
& (\nabla R i c)_{x}\left(F_{x}\left(\partial_{\tilde{v}}\right)_{x_{o}}, F_{x}\left(\partial_{\tilde{v}}\right)_{x_{o}}, F_{x}\left(\partial_{\tilde{v}}\right)_{x_{o}}\right)=\iota_{o}^{\prime},
\end{aligned}
$$

where $a d \neq 0$. Therefore we have

$$
\begin{aligned}
& d^{2} \iota=\iota_{o}, \\
& d^{3} \iota^{\prime}=\iota_{o}^{\prime}
\end{aligned}
$$

at every point of $U$. Suppose that $\nabla$ is not locally symmetric. Then, by the curvature homogeneity of order $1, \iota^{\prime} \neq 0$ everywhere. Also $\iota_{o} \neq 0$. Moreover $d$ is a nowhere vanishing smooth function of one variable $v$. Differentiating (1.6) yields

$$
2 d d^{\prime} \iota+d^{2} \iota^{\prime}=0 .
$$

Mulitiplying (1.8) by $d$ and using (1.6) and (1.7) we obtain

$$
2 d^{\prime} \iota_{o}+\iota_{o}^{\prime}=0 .
$$

Hence $d^{\prime \prime}=0$ and consequently, by (1.6), we have $\iota(v)=\epsilon(q v+p)^{-2}$ for some numbers $q \neq 0, p$ and $\epsilon=1$ or -1 . The same applies to $\tilde{\iota}$, i.e. there are numbers $\tilde{q} \neq 0$ and $\tilde{p}$ such that $\tilde{\iota}(\tilde{v})=\epsilon(\tilde{q} \tilde{v}+\tilde{p})^{-2}$. We have

$$
\iota^{(k)}(v)=\epsilon(-1)^{k}(k+1) ! q^{k}(q v+p)^{-(k+2)}
$$

and

$$
\iota_{o}^{(k)}=\epsilon(-1)^{k}(k+1) ! \tilde{q}^{k}\left(\tilde{q} v_{o}+\tilde{p}\right)^{-(k+2)} .
$$

Formula (1.6) implies that $d(v)=(q v+p)\left(\tilde{q} \tilde{v}_{o}+\tilde{p}\right)^{-1}$. Therefore, by (1.7), (1.9) and (1.10), we have $\tilde{q}=q$. Using (1.9) and (1.10) it is now easy to see that

$$
d^{k+2} \iota^{(k)}=\iota_{o}^{(k)}
$$

for every $k$. This is equivalent to the equality

$$
\left(\nabla^{k} R i c\right)\left(F_{x}\left(\partial_{\tilde{v}}\right)_{x_{o}}, \ldots, F_{x}\left(\partial_{\tilde{v}}\right)_{x_{o}}\right)=\left(\nabla^{k} R i c\right)\left(\left(\partial_{\tilde{v}}\right)_{x_{o}}, \ldots,\left(\partial_{\tilde{v}}\right)_{x_{o}}\right)
$$

for every $k$. Using Theorem 7.2 from [1] completes the proof.

A consequence of the above proof and Lemma 1.1 is the following 
Corollary 1.3. Let $\nabla$ be a projectively flat connection on a 2-dimensional manifold $M$. Assume that the Ricci tensor of $\nabla$ has rank 1 and the distribution ker Ric is $\nabla$-parallel. If $\nabla$ is locally homogeneous, then for every canonical coordinate system $(u, v)$ for $\nabla$ the function $\iota=\operatorname{Ric}\left(\partial_{v}, \partial_{v}\right)$ is given by

$$
\iota(v)=\epsilon(q v+p)^{-2}
$$

for some numbers $q$ and $p$ and $\epsilon= \pm 1$.

Corollary 1.4. Let $\nabla$ be an analytic projectively flat connection on a 2-dimensional analytic manifold $M$. Assume that the Ricci tensor of $\nabla$ has rank 1 and the distribution ker Ric is $\nabla$-parallel. The connection $\nabla$ is locally homogeneous and not locally symmetric if and only if for every $x \in M$ there is a coordinate system $(u, v)$ around $x$ such that

$$
\nabla_{\partial_{u}} \partial_{u}=\nabla_{\partial_{u}} \partial_{v}=0, \quad \nabla_{\partial_{v}} \partial_{v}=\epsilon u(q v+p)^{-2} \partial_{u}
$$

for some numbers $q \neq 0, p$ and $\epsilon= \pm 1$.

Proof. Assume that $\nabla$ is locally homogeneous and not locally symmetric. Let $(u, v)$ be a canonical coordinate system for $\nabla$ around $x$. By Corollary 1.3 we have $\iota=\epsilon(q v+p)^{-2}$. If $q=0$, then $\nabla$ is locally symmetric. Hence we can assume that $q>0$. Take the following connection $\tilde{\nabla}$ on $\mathbf{R} \times\left(-\frac{p}{q}, \infty\right)$ :

$$
\tilde{\nabla}_{\partial_{\tilde{u}}} \partial_{\tilde{u}}=\tilde{\nabla}_{\partial_{\tilde{u}}} \partial_{\tilde{v}}=0, \quad \tilde{\nabla}_{\partial_{\tilde{v}}} \partial_{\tilde{v}}=\epsilon \tilde{u}(q \tilde{v}+p)^{-2} \partial_{\tilde{u}},
$$

where $(\tilde{u}, \tilde{v})$ is the standard coordinate system on $\mathbf{R}^{2}$. Let $F_{x}: T_{x} M \rightarrow T_{\left(0,-\frac{p}{q}+\frac{1}{q}\right)} \mathbf{R}^{2}$ be the linear isomorphism given by

$$
F_{x}\left(\partial_{u}\right)_{x}=E_{1}, \quad F_{x}\left(\partial_{v}\right)_{x}=(q v+p)^{-1} E_{2},
$$

where $E_{1}, E_{2}$ is the canonical basis of $\mathbf{R}^{2}$. Similarly as in the proof of Theorem 1.2 , one can check that $F_{x}$ transforms $\nabla^{k}$ Ric into $\tilde{\nabla}^{k} \tilde{R} i c$ for every $k$, where $\tilde{R} i c$ is the Ricci tensor for $\tilde{\nabla}$. By Theorem 7.2 from [1] $F_{x}$ can be extended to an affine isomorphism around $x$. The isomorphism gives a desired coordinate system.

Similarly as above, one can prove that if $\nabla$ is locally symmetric and its Ricci tensor has rank 1 , then for every $x \in M$ there is a coordinate system $(u, v)$ around $x$ in which $\nabla$ is given by

$$
\nabla_{\partial_{u}} \partial_{u}=\nabla_{\partial_{u}} \partial_{v}=0, \quad \nabla_{\partial_{v}} \partial_{v}=\epsilon u \partial_{u}
$$

where $\epsilon=1$ or -1 . Using Lemma 1.1 one can find many projectively flat connections whose Ricci tensor has rank 1 . As it has been shown, most of them are not locally homogeneous. For instance, let $(u, v)$ be any coordinate system on a manifold $M$ and $\phi(v)$ be an arbitrary function different than $\epsilon(q v+p)^{-2}$ for any numbers $q, p$. The following connection is projectively flat curvature homogeneous and not locally homogeneous:

$$
\nabla_{\partial_{u}} \partial_{u}=\nabla_{\partial_{u}} \partial_{v}=0, \quad \nabla_{\partial_{v}} \partial_{v}=u \phi(v) \partial_{u} .
$$

Among the connections given by (1.14) there are complete ones. For instance, take $\nabla$ given by (1.14) on $\mathbf{R}^{2}$ where $\phi(v)=-2\left(1+v^{2}\right)^{-1}$. Then straight lines parallel to the the axis $u$ and curves

$$
\mathbf{R} \ni t \rightarrow\left(\alpha\left(1+t^{2}\right)+\frac{\beta}{2}(t+\operatorname{arctg} t), t\right)
$$

for $\alpha, \beta \in \mathbf{R}$ are all pregeodesics of $\nabla$. 
In this section we shall give other examples of projectively flat locally homogeneous connections which are not locally symmetric. If for an analytic connection $\nabla$ on a 2-dimensional manifold $M$ there exist constant numbers $A, B, C, D, E, F$, $G, H$ such that for every $x \in M$ there is a frame $X, Y$ around $x$ relative to which $\nabla$ is given by

$\nabla_{X} X=A X+B Y, \quad \nabla_{X} Y=C X+D Y, \quad \nabla_{Y} X=E X+F Y, \quad \nabla_{Y} Y=G X+H Y$, then $\nabla$ is locally homogeneous. Consider the following projectively flat connection on $\mathbf{R}^{2} \backslash\{u=0\}$ where $(u, v)$ is the canonical coordinate system on $\mathbf{R}^{2}$ :

$$
\nabla_{\partial_{u}} \partial_{u}=0, \quad \nabla_{\partial_{u}} \partial_{v}=\frac{1}{u} \partial_{v}, \quad \nabla_{\partial_{v}} \partial_{v}=u^{2} \partial_{u}
$$

Take the frame $X, Y$ :

$$
X=u \partial_{u}, \quad Y=u^{-\frac{1}{2}} \partial_{v}
$$

Then

$$
\nabla_{X} X=X, \quad \nabla_{X} Y=\frac{1}{2} Y, \quad \nabla_{Y} X=Y, \quad \nabla_{Y} Y=X .
$$

Hence the connection is locally homogeneous. The connection has Ricci tensor of rank 1. The connection (2.1) has been found as a projectively flat one which cannot be realized, even locally, on any non-degenerate surface in $\mathbf{R}^{3}$. It can be realized on cones. There also exist locally homogeneous projectively flat non-locally symmetric connections whose Ricci tensor is non-degenerate. For instance, consider the following connection on $\mathbf{R}^{2}$ :

$$
\begin{aligned}
\nabla_{\partial_{u}} \partial_{u} & =C \partial_{u}+D \partial_{v}, \\
\nabla_{\partial_{u}} \partial_{v} & =0, \\
\nabla_{\partial_{v}} \partial_{v} & =A \partial_{u}+B \partial_{v},
\end{aligned}
$$

where $A, B, C, D$ are numbers such that $A D \neq 0$ and $A D-C B \neq 0$. The connection is locally homogeneous, projectively flat and not locally symmetric. Its Ricci tensor is symmetric and non-degenerate.

\section{REFERENCES}

1. S. Kobayashi and K. Nomizu, Foundations of Differential Geometry, vol. I, Interscience Publishers, New York and London, 1963. MR 27:2945

2. B. Opozda, Locally symmetric connections on surfaces, Results in Math. 20 (1991), 725-743. CMP 92:13

3. _ A class of projectively flat surfaces, Math. Z. 219 (1995), 77-92.

4. B. Opozda and T. Sasaki, Surfaces whose images of the affine normal are curves, Kyushu J. Math. 49 (1995), 1-10.

5. I. M. Singer, Infinitesimaly homogeneous spaces, Comm. Pure Appl. Math. 13 (1960), 685-697. MR 24:A1100

Institute of Mathematics, Jagiellonian University, Reymonta 4, 30-059, Kraków, POLAND

E-mail address: opozda@im.uj.edu.pl 